\title{
EEG correlates of strategies of emotional regulation during perception of emotional information
}

\author{
Andrey V. Bocharov \\ Laboratory of Differential \\ Psychophysiology, Scientific \\ Research Institute of Physiology \\ and Basic Medicine, Novosibirsk, \\ Russia \\ Humanitarian Institute, Novosibirsk \\ State University, Novosibirsk, \\ Russia \\ Alexander N. Savostyanov \\ Laboratory of Differential \\ Psychophysiology, Scientific \\ Research Institute of Physiology \\ and Basic Medicine, Novosibirsk, \\ Russia, Humanitarian Institute, \\ Novosibirsk State University, \\ Novosibirsk, Russia \\ Institute of Cytology and Genetics \\ SB RAS, Novosibirsk, Russia
}

\author{
Sergey S. Tamozhnikov \\ Laboratory of Differential \\ Psychophysiology, Scientific \\ Research Institute of Physiology \\ and Basic Medicine, Novosibirsk, \\ Russia
}

Ekaterina A. Merkulova2

Laboratory of Differential

Psychophysiology, Scientific

Research Institute of Physiology and Basic Medicine, Novosibirsk, Russia

\author{
Alexander E. Saprigyn \\ Laboratory of Differential \\ Psychophysiology, Scientific \\ Research Institute of Physiology \\ and Basic Medicine, Novosibirsk, \\ Russia
}

\author{
Ekaterina A. Proshina \\ Laboratory of Differential \\ Psychophysiology, Scientific \\ Research Institute of Physiology \\ and Basic Medicine, Novosibirsk, \\ Russia \\ Gennady G. Knyazev \\ Laboratory of Differential \\ Psychophysiology, Scientific \\ Research Institute of Physiology \\ and Basic Medicine, Novosibirsk, \\ Russia
}

\begin{abstract}
The aim of research was to study the relation between oscillatory dynamics during the perception of negative, positive and neutral sentences and emotional facial expressions and strategies of perception and processing emotional information.
\end{abstract}

Keywords - EEG, reappraisal, suppression, emotional sentences, emotional facial expressions

72 subjects (43 women) took part in EEG study and completed J. Gross questionnaire (2003). In task of the searching of syntax errors, the participants were needed to determine whether the sentence contains a syntax error or not in three types of emotional colored sentences (negative, neutral and positive).

Four types of facial emotional expressions (angry, neutral, sad, and happy) were used. EEG recording was performed using a cap with 127 electrodes. To measure the induced responses, event-related spectral perturbations (ERSP) were calculated.

"Reappraisal emotions" scale positively correlated with an increase in alpha spectral power in the first 200 ms after the presentation of sad facial expressions. According to the Klimesch hypothesis (2011), early alpha oscillations in response to visual stimuli may be associated with inhibition, which is effective during initial access to a complex knowledge system. It could be suggested that an early increase in alpha oscillations in subjects who prefer a strategy of cognitive reappraisal of emotions could reflect a preparation for the perception of emotional information.

Reappraisal emotions positively correlated with an increase in theta rhythm in the time interval from 200 to $400 \mathrm{~ms}$ after presentation of negative sentences which described symptoms of depression and after presentation of angry faces. It could be assumed that people who prefer the strategy of Reappraisal emotions, during perception of negative information, pay more attention to the emotional content of stimuli. Emotion suppression scale was positively correlated with ERSP of beta range after presentation of positive sentences which contained syntax errors. It could be related with a decrease of top-down control processes and a decrease of semantic processes during the search of syntax errors in positive sentences.

\section{ACKNOWLEDGMENT}

The study was supported by the Russian Foundation for Basic Research (RFBR) under Grants No. 18-313-00174, No. 18-00-00939, No. 20-01300404 , No. 18-29-13027 and partially supported by the federal budget for basic scientific research theme No. AAAA-A16-116021010228-0. 